\title{
Permanent pacing for cardioinhibitory malignant vasovagal syndrome
}

M E V Petersen, R Chamberlain-Webber, A P Fitzpatrick, A Ingram, T Williams, R Sutton

Chelsea and

Westminster Hospital, London

M E V Petersen

R Chamberlain-Webber

A P Fitzpatrick

A Ingram

T Williams

$R$ Sutton

Correspondence to:

Dr Richard Sutton

Department of Cardiology,

Chelsea and Westminster

Hospital, 369 Fulham Road,

London SW 10 9NH.

Accepted for publication

6 September 1993
Abstract

Objective-To evaluate the effect of permanent pacing in cardioinhibitory malignant vasovagal syndrome.

Patients and methods-37 patients with permanent pacemakers for cardioinhibitory malignant vasovagal syndrome. All presented with syncope (median six episodes, median frequency two episodes a year) and after conventional investigation and invasive electrophysiological assessment they remained undiagnosed, and without a generally accepted indication for pacemaker implantation. In all vasovagal syncope with cardioinhibition (heart rate at syncope $<60$ beats $/ \mathrm{min}$ ) developed during tilt tests performed according to the Westminster protocol (head up tilt at $60^{\circ}$ with a footplate support for 45 minutes or until syncope intervenes). Dual chamber pacemakers were implanted in $35(95 \%)$ and VVI pacemakers in the remaining two (5\%).

Results-Over a mean (SD) follow up since implantation of 50.2 (23.9) months symptomatic improvement occurred in $89 \%$ : $62 \%$ remained free of syncope and $27 \%$ were completely symptom free. The collective syncopal burden of these 37 patients was reduced from 136 to 11 episodes each year. During follow up three patients died from unrelated causes. Patients who become asystolic during the tilt test (sinus pause of at least four seconds) experienced no greater benefit from pacing than those with less extreme cardioinhibition. Patients who remained free of syncope since implantation were younger than those who continued to experience syncope. Patients who remained completely symptom free after implantation were younger, more likely to be male, and had had fewer syncopal episodes before implantation than those who continued to experience syncope or presyncope. No other demographic, clinical, investigative, or pacing variable suggested a more favourable outcome after implant.

Conclusions-This retrospective and uncontrolled experience suggests a possible role for permanent pacing in selected patients with cardioinhibitory malignant vasovagal syndrome. Improved acquisition of tilt test data may enable better selection of patients who are suitable for permanent pacing. A randomised prospective study to compare permanent pacing with no treatment or with medical treatment in cardioinhibitory malignant vasovagal syndrome is indicated.

(Br Heart f 1994;71:274-281)

Recurrent syncope is a common clinical problem which, despite extensive investigation, remains unexplained in $30 \%-50 \%$ of patients. ${ }^{1-4}$ In 1986 Kenny et al reported a series of 15 patients with unexplained syncope who had been subjected to prolonged head up tilt at $40^{\circ}$ for up to 60 minutes. ${ }^{5}$ Ten $(67 \%)$ of this group developed vasovagal syncope during tilt by contrast with one $(10 \%)$ of 10 age matched controls. The authors suggested that vasovagal syncope was the cause of these patients' clinical events and that a prolonged head up tilt test was a useful test in the investigation of unexplained syncope. Subsequently, tilt testing in this context has been widely evaluated, the original findings reproduced, ${ }^{4-9}$ and the conclusions of Kenny et al are now generally accepted. ${ }^{4-12}$

The nature of the predisposition to vasovagal syncope identified by tilt tests may vary between subjects and has been incompletely established. ${ }^{12-14}$ Therefore, at present, patients with otherwise unexplained syncope and positive tilt tests are best considered clinically as having malignant vasovagal syndrome. ${ }^{15} 16$ The term malignant has been included to indicate that the presentation is of frequent or severe syncopal attacks that may be clinically difficult to distinguish from Adams-Stokes syncope. ${ }^{1516}$ This differentiates these subjects from those with more predictable, ${ }^{17}$ infrequent, and less severe vasovagal episodes, usually associated with a longer prodrome, and most prevalent in young people. ${ }^{13}$

While the pathophysiology of vasovagal syncope remains incompletely understood, ${ }^{13}$ appropriate treatment remains uncertain, ${ }^{18}$ and pacing treatment controversial. ${ }^{1019} \mathrm{We}$ report our retrospective experience of symptomatic management with permanent pacing in 37 patients with cardioinhibitory malignant vasovagal syndrome.

\section{Patients and methods}

PATIENT SELECTION

The study group included all patients who have received permanent pacemakers at the Westminster Hospital for cardioinhibitory malignant vasovagal syndrome before 1992 . 
The clinical and pacemaker records and the results of investigations were reviewed for all patients who had positive tilt tests and subsequently received a permanent pacemaker.

Table 1 Clinical characteristics (all patients)

\begin{tabular}{|c|c|c|c|c|c|c|c|}
\hline $\begin{array}{l}\text { Patient } \\
\text { No } \\
\end{array}$ & Age & Sex & Syncope-n & Syncope-freq & Prodrome & Injury & Electrocardiogram \\
\hline 1 & 68 & F & 10 & 5 & $\mathrm{Y}$ & $\mathbf{Y}$ & SB, INFMI \\
\hline 2 & 73 & $\mathbf{M}$ & 2 & 2 & $\mathbf{Y}$ & $\mathbf{Y}$ & \\
\hline 3 & 66 & $\mathbf{F}$ & 110 & 2 & $\mathrm{Y}$ & $\mathbf{N}$ & Normal \\
\hline 4 & 66 & M & 1 & $\overline{1}$ & $\bar{Y}$ & $\mathbf{N}$ & Normal \\
\hline 5 & 38 & $\mathbf{F}$ & 70 & 25 & $\mathrm{Y}$ & $\mathrm{N}$ & \\
\hline 6 & 78 & $\mathrm{~F}$ & 4 & 0.5 & $\mathrm{Y}$ & $\mathbf{N}$ & Normal \\
\hline 7 & 42 & M & 1 & 1 & $\dot{\mathrm{Y}}$ & $\mathbf{N}$ & \\
\hline 8 & 65 & $\mathbf{F}$ & 3 & 1.5 & $\mathrm{Y}$ & $\mathrm{Y}$ & RBBB \\
\hline 9 & 74 & $\mathbf{F}$ & 10 & 0.5 & $\mathbf{Y}$ & $\mathbf{N}$ & $\mathrm{AF}$ \\
\hline 10 & 46 & $\mathbf{M}$ & 3 & 6 & $\mathbf{Y}$ & $\mathbf{Y}$ & \\
\hline 11 & 53 & $\mathbf{F}$ & 6 & 6 & $\bar{Y}$ & $\mathbf{N}$ & Normal \\
\hline 12 & 89 & $\mathbf{F}$ & 6 & 1 & $\mathbf{N}$ & $\mathrm{Y}$ & Normal \\
\hline 13 & 33 & $\mathbf{M}$ & 4 & 12 & $\mathrm{Y}$ & $\mathbf{N}$ & Normal \\
\hline 14 & 53 & M & 6 & 0.4 & $\mathbf{Y}$ & $\mathbf{N}$ & Normal \\
\hline 15 & 52 & M & 4 & 3 & $\mathbf{N}$ & $\mathrm{Y}$ & Normal \\
\hline 16 & 74 & M & 6 & 0.5 & $\mathrm{Y}$ & $\mathbf{Y}$ & SB, LAFB \\
\hline 17 & 73 & $\mathbf{F}$ & 1 & 0.2 & $\mathbf{N}$ & $\mathbf{N}$ & Normal \\
\hline 18 & 73 & $\mathbf{F}$ & 6 & 1 & $\mathrm{Y}$ & $\mathbf{N}$ & \\
\hline 19 & 36 & M & 25 & 1 & $\mathbf{Y}$ & $\mathbf{N}$ & Normal \\
\hline 20 & 48 & $\mathbf{F}$ & 60 & 3 & $\mathbf{Y}$ & $\mathbf{N}$ & Normal \\
\hline 21 & 66 & $\mathbf{F}$ & 20 & 10 & $\mathbf{Y}$ & $\mathbf{N}$ & Normal \\
\hline 22 & 59 & $\mathbf{M}$ & 14 & 0.7 & $\mathbf{Y}$ & $\mathrm{Y}$ & Normal \\
\hline 23 & 69 & M & 2 & 2 & $\bar{Y}$ & $\mathbf{N}$ & \\
\hline 24 & 82 & $\mathbf{F}$ & 15 & 3 & $\hat{\mathbf{Y}}$ & $\mathbf{N}$ & $\mathrm{ST} / \mathrm{T}$ ABS \\
\hline 25 & 47 & $\mathbf{M}$ & 1 & 1 & $\mathbf{Y}$ & $\mathbf{N}$ & Normal \\
\hline 26 & 84 & $\mathbf{F}$ & 15 & 3 & $\mathbf{N}$ & $\mathbf{N}$ & LAFB \\
\hline 2 & 51 & $\mathbf{M}$ & 2 & $2 \cdot 6$ & $\mathrm{Y}$ & $\mathbf{N}$ & Normal \\
\hline 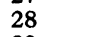 & 63 & M & 50 & 12 & $\dot{\mathbf{Y}}$ & $\mathbf{N}$ & INFMI \\
\hline 29 & 79 & $\mathrm{~F}$ & 2 & 4 & $\overline{\mathbf{Y}}$ & $\mathbf{N}$ & Normal \\
\hline 30 & 41 & $\hat{\mathrm{F}}$ & 12 & 4 & $\mathbf{N}$ & $\mathrm{Y}$ & Normal \\
\hline 31 & 37 & $\mathbf{M}$ & 20 & $1 \cdot 4$ & $\mathbf{Y}$ & $\mathbf{N}$ & \\
\hline 32 & 71 & $\mathbf{M}$ & 6 & 2 & $\mathbf{N}$ & $\mathrm{Y}$ & Normal \\
\hline 33 & 66 & $\mathbf{M}$ & 6 & 0.7 & $\mathbf{Y}$ & $\mathbf{N}$ & Normal \\
\hline 34 & 62 & M & 1 & 1 & $\mathbf{N}$ & $\mathbf{N}$ & Normal \\
\hline 35 & 84 & $\mathbf{M}$ & 40 & 20 & $\mathbf{Y}$ & $\mathrm{Y}$ & AVB-1 \\
\hline 36 & 38 & $\mathbf{M}$ & 1 & 1 & $\mathbf{Y}$ & $\mathbf{N}$ & Normal \\
\hline 37 & 58 & $\mathrm{M}$ & 2 & 0.7 & $\mathbf{N}$ & $\mathbf{N}$ & LAFB \\
\hline
\end{tabular}

Syncope-n, total number of reported episodes of syncope; Syncope-freq, frequency of syncope (episodes/year); Prodrome, symptomatic period preceding syncope; Injury, injury as a result of syncopal episode; SB, sinus bradycardia; LAFB, left anterior fasicular block; INFMI, inferior myocardial infarction; AF, atrial fibrillation; RBBB, right bundle branch block; AVB-1, prolonged PR interval; ST/T ABS, non-specific repolarisation abnormality; $Y$, yes; N, no.

\section{Table 2 Tilt test results (all patients)}

\begin{tabular}{|c|c|c|c|c|c|c|c|c|c|c|c|}
\hline $\begin{array}{l}\text { Patient } \\
\text { No }\end{array}$ & $\begin{array}{l}\text { Tilts } \\
(n)\end{array}$ & $P$ & $N$ & Tilt1 & Tilt2 & $\begin{array}{l}\text { Time } \\
\text { (min) }\end{array}$ & $H R s$ & $H R \max$ & $\mathrm{HRe}$ & BPSYSS & $B P D L A s$ \\
\hline 1 & 1 & 1 & 0 & $\mathbf{P}$ & - & 25 & 78 & 79 & 20 & 143 & 98 \\
\hline 2 & 1 & 1 & 0 & $P$ & - & $45^{\star}$ & 40 & 68 & 7 & 167 & 85 \\
\hline 3 & 1 & 1 & 0 & $\mathbf{P}$ & - & 21 & 74 & 80 & 43 & 165 & 82 \\
\hline 4 & 2 & 2 & 0 & $\mathbf{P}$ & $\mathbf{P}$ & 15 & 60 & 73 & 42 & 153 & 90 \\
\hline 5 & 1 & 1 & 0 & $\mathbf{P}$ & - & 27 & 60 & 105 & 42 & 112 & 74 \\
\hline 6 & 2 & 2 & 0 & $P$ & $\mathbf{P}$ & 13 & 76 & 84 & 45 & 143 & 77 \\
\hline 7 & 2 & 2 & 0 & $\mathbf{P}$ & $\mathbf{P}$ & 4 & 49 & 64 & 30 & 126 & 68 \\
\hline 8 & 2 & 2 & 0 & $\mathbf{P}$ & $\mathbf{P}$ & 35 & 66 & 104 & 20 & 113 & 86 \\
\hline 9 & 1 & 1 & 0 & P & - & 28 & 75 & 85 & 33 & 157 & 100 \\
\hline 10 & 5 & 2 & 3 & $\mathbf{N}$ & $\mathbf{N}$ & 23 & 41 & 53 & 12 & 113 & 71 \\
\hline 11 & 2 & 1 & 1 & $\mathbf{N}$ & $\mathbf{P}$ & 18 & 74 & 95 & 44 & 170 & 90 \\
\hline 12 & 1 & 1 & 0 & $\mathbf{P}$ & - & 18 & 70 & 76 & 45 & 158 & 90 \\
\hline 13 & 2 & 2 & 0 & $\mathbf{P}$ & $\mathbf{P}$ & 18 & 58 & 66 & 6 & 130 & 70 \\
\hline 14 & 1 & 1 & 0 & $\mathbf{P}$ & - & 20 & 51 & 56 & 8 & 142 & 71 \\
\hline 15 & 1 & 1 & 0 & $P$ & - & 27 & 74 & 89 & 27 & 164 & 104 \\
\hline 16 & 2 & 2 & 0 & P & $\mathbf{P}$ & 40 & 69 & 89 & 35 & 220 & 107 \\
\hline 17 & 1 & 1 & 0 & $\mathbf{P}$ & - & 20 & 70 & 82 & 4 & 125 & 80 \\
\hline 18 & 1 & 1 & 0 & $\mathbf{P}$ & - & 11 & 58 & 68 & 39 & 156 & 64 \\
\hline 19 & 2 & 2 & 0 & $\mathbf{P}$ & $\mathbf{P}$ & 1 & 80 & 110 & 1 & 120 & 80 \\
\hline 20 & 3 & 1 & 2 & $\mathbf{N}$ & $\mathbf{N}$ & 8 & 76 & 115 & 3 & 130 & 83 \\
\hline 21 & 1 & 1 & 0 & $P$ & - & 12 & 60 & 80 & 32 & 180 & 100 \\
\hline 22 & 1 & 1 & 0 & $P$ & - & 25 & 60 & 67 & 44 & 120 & 63 \\
\hline 23 & 2 & 1 & 1 & $\mathbf{N}$ & $\mathbf{P}$ & $47^{\star}$ & 61 & 68 & 44 & 145 & 93 \\
\hline 24 & 1 & 1 & 0 & $P$ & - & 35 & 64 & 64 & 3 & 139 & 69 \\
\hline 25 & i & 1 & 0 & $P$ & - & 22 & 67 & 85 & 58 & 133 & 73 \\
\hline 26 & 1 & 1 & 0 & $\mathbf{P}$ & - & $55^{\star}$ & 83 & 100 & 44 & 160 & 90 \\
\hline 27 & 1 & 1 & 0 & $\mathbf{P}$ & - & 8 & 77 & 80 & 56 & 137 & 102 \\
\hline 28 & 3 & 2 & 1 & $\mathbf{P}$ & $\mathbf{P}$ & 15 & 75 & 82 & 30 & 160 & 75 \\
\hline 29 & 1 & 1 & 0 & $\mathbf{P}$ & - & 22 & 80 & 90 & 12 & 105 & 50 \\
\hline 30 & 3 & 1 & 2 & $\mathbf{N}$ & $\mathbf{N}$ & 22 & 75 & 120 & 59 & 95 & 55 \\
\hline 31 & 1 & 1 & 0 & $\mathbf{P}$ & - & 35 & 57 & 86 & 41 & 128 & 83 \\
\hline 32 & 2 & 2 & 0 & $\mathbf{P}$ & $\mathbf{P}$ & 16 & 70 & 75 & 53 & 142 & 73 \\
\hline 33 & 1 & 1 & 0 & $\mathbf{P}$ & - & 45 & 51 & 87 & 7 & 159 & 90 \\
\hline 34 & 2 & 1 & 1 & $\mathbf{P}$ & $\mathbf{N}$ & 37 & 70 & 109 & 58 & 170 & 100 \\
\hline 35 & 3 & 1 & 2 & $\mathbf{P}$ & $\mathbf{N}$ & 12 & 58 & 65 & 32 & 158 & 68 \\
\hline 36 & 1 & 1 & 0 & $\mathbf{P}$ & - & 3 & 63 & 74 & 34 & 105 & 70 \\
\hline 37 & 1 & 1 & 0 & $\mathbf{P}$ & - & 4 & 83 & 89 & 14 & 146 & 91 \\
\hline
\end{tabular}

*Tilt performed for 60 minutes before change in method. Tilts total number of tilt tests before implantation; $\mathrm{P}$, positive; N, negative; Time, time of positive tilt; HRs, resting heart rate; HRmax, maximum heart rate; HRe, minimum heart rate at syncope; BPSYSs, resting systolic blood pressure; BPDIAs, resting diastolic blood pressure.
Inclusion criteria were a history of syncope and a syncopal outcome with bradycardia (heart rate $<60$ at syncope) during tilt tests performed according to the Westminster Hospital protocol.

Patients were excluded from analysis if any evidence of second or third degree atrioventricular block, sinus node disease, carotid sinus hypersensitivity, tachyarrhythmia, or obstructive cardiac pathology had been found during clinical assessment, conventional investigation, and a limited invasive electrophysiological study.

Resting electrocardiography and 24 hour ambulatory electrocardiography were performed in all patients. Exercise tests were performed in $51 \%$ and transthoracic echocardiography in $22 \%$ when clinically indicated. A limited electrophysiological study, conducted in the resting postabsorptive nonsedated state, and carotid sinus massage were performed in all patients. No patient showed prolonged intracardiac conduction times (AH > $120 \mathrm{~ms}, \mathrm{HV}>60 \mathrm{~ms}$ ), an anterograde Wenckebach point $<120$ beats/min during incremental atrial pacing, a corrected sinus node recovery time $>700 \mathrm{~ms}$ at multiple atrial pacing rates or a ventricular pause of three seconds or more during carotid sinus massage for six seconds. Thirty two per cent of the patients were subjected to a ventricular extrastimulation study and none developed a sustained tachycardia.

The Westminster Hospital tilt test protocol has been previously reported. ${ }^{20}$ Prolonged head up tilt is conducted at $60^{\circ}$ on a foot plate support for up to 45 minutes or until syncope intervenes. Patients are monitored non-invasively with a continuous electrocardiogram and intermittent (Dinamap, Critikon, USA) or continuous (Finapress, Ohmeda, Englewood, CO, USA) blood pressure measurements. The tilt table (G Shaw and Sons Ltd, Nottingham, England) achieves $60^{\circ}$ tilt at $4 \%$. Patients are restrained during tilt with two velcro straps placed around the lower legs and waist. The test is performed without conversation in subdued lighting.

\section{PACEMAKER MODE AND PROGRAMMING}

\section{SELECTIONS}

Dual chamber pacing had been selected for all but two patients (one with atrial fibrillation) to achieve maximum haemodynamic benefit during support of bradycardia, and minimise the occurrence of pacemaker syndrome. Most patients (84\%) had been initially paced in DDI mode with rate hysteresis. The DDI mode excludes the possibility of pacemaker mediated tachycardia. Rate hysteresis with a trigger rate selected below the subjects sleeping heart rate avoids unnecessary pacing under normal circumstances and allows a higher rate of pacing support when required.

\section{COLLECTION OF DATA}

For all patients complete records of demographic details, symptoms before 
Table 3 Follow up data (all patients)

\begin{tabular}{|c|c|c|c|c|c|c|c|c|c|c|}
\hline $\begin{array}{l}\text { Patient } \\
\text { No }\end{array}$ & $\begin{array}{l}F U \\
\text { (months) }\end{array}$ & $S-f u-n$ & $S$-freq-fu & $P S-f u$ & Mode-1 & Mode-c & Mode-c- $t$ & Mode-c-s & Mode-c-np & Mode-final \\
\hline 1 & 87 & 1 & $0 \cdot 14$ & $\mathrm{Y}$ & DDI $85 / 45$ & 4 & Yt & $\mathrm{Y}$ & $\mathrm{Y}$ & AAI $80 / 40$ \\
\hline 2 & 61 & 4 & 0.78 & $\mathrm{Y}$ & DDD 60 & 0 & $\mathrm{~N}$ & $\mathbf{N}$ & $\mathbf{N}$ & DDD 60 \\
\hline 3 & 74 & 3 & 0.49 & $\mathrm{Y}$ & DDI $85 / 45$ & 1 & $\mathrm{~N}$ & $\mathbf{N}$ & $\mathrm{Y}$ & DDI $80 / 40$ \\
\hline 4 & 56 & 0 & 0 & $\mathbf{N}$ & DDI $80 / 50$ & 1 & $\mathbf{N}$ & $\mathbf{N}$ & $\mathrm{Y}$ & DDI $80 / 40$ \\
\hline 5 & 37 & 0 & 0 & $\mathrm{Y}$ & DDI $80 / 40$ & 2 & $\mathrm{~N}$ & $\mathbf{Y}$ & $\dot{Y}$ & DDI $80 / 45$ \\
\hline 6 & 35 & 2 & 0.69 & $\mathrm{Y}$ & DDI $80 / 55$ & 0 & $\mathrm{~N}$ & $\mathbf{N}$ & $\mathbf{N}$ & DDI $90 / 50$ \\
\hline 7 & 82 & 0 & 0 & $\mathrm{~N}$ & DDI $85 / 45$ & 2 & $\mathbf{N}$ & $\mathrm{N}$ & $\mathrm{Y}$ & DDI $60 / 45$ \\
\hline 8 & 61 & 0 & 0 & $\mathrm{~N}$ & DDI $80 / 40$ & 1 & $\mathrm{~N}$ & $\mathrm{~N}$ & $\mathrm{Y}$ & DDI $70 / 40$ \\
\hline 9 & 33 & 0 & 0 & $\mathrm{Y}$ & VVI 70 & 0 & $\mathrm{~N}$ & $\mathrm{~N}$ & $\mathbf{N}$ & VVI 70 \\
\hline 10 & 16 & 1 & 0.75 & $\mathrm{Y}$ & DDD 60 & 1 & $\mathbf{N}$ & $\mathrm{Y}$ & $\mathbf{N}$ & DDD 70 \\
\hline 11 & 65 & 4 & 0.74 & $\mathrm{Y}$ & DDI $80 / 50$ & 2 & $\mathbf{N}$ & $\mathrm{Y}$ & $\mathbf{N}$ & DDI $90 / 55$ \\
\hline 12 & $21^{\star}$ & 0 & 0 & $\mathrm{Y}$ & DDI $85 / 45$ & $\overline{1}$ & $\mathbf{N}$ & $\mathrm{Y}$ & $\mathbf{N}$ & DDI $85 / 50$ \\
\hline 13 & 14 & 0 & 0 & $\mathrm{~N}$ & DDD 60 & 0 & $\mathrm{~N}$ & $\mathrm{~N}$ & $\mathrm{~N}$ & DDD 60 \\
\hline 14 & 50 & 0 & 0 & $\mathrm{Y}$ & DDI 90/55 & 3 & $\mathbf{N}$ & $\mathrm{Y}$ & $\mathrm{Y}$ & DDI $75 / 45$ \\
\hline 15 & 62 & 0 & 0 & $\mathrm{Y}$ & DDI $80 / 50$ & 0 & $\mathrm{~N}$ & $\mathbf{N}$ & $\mathbf{N}$ & DDI $80 / 50$ \\
\hline 16 & 42 & 2 & 0.58 & $\mathrm{Y}$ & DDD 60 & 0 & $\mathrm{~N}$ & $\mathrm{~N}$ & $\mathbf{N}$ & DDD 60 \\
\hline 17 & 77 & 0 & 0 & $\mathrm{Y}$ & DDI $90 / 50$ & 2 & $\mathrm{~N}$ & $\mathrm{Y}$ & $\mathrm{Y}$ & DDI $80 / 50$ \\
\hline 18 & 49 & 1 & $0 \cdot 25$ & $\mathrm{Y}$ & DDI $90 / 50$ & 0 & $\mathbf{N}$ & $\mathbf{N}$ & $\mathrm{N}$ & DDI $90 / 50$ \\
\hline 19 & 11 & 0 & 0 & $\mathrm{~N}$ & DDI $85 / 45$ & 0 & $\mathrm{~N}$ & $\mathrm{~N}$ & $\mathrm{~N}$ & DDI $85 / 45$ \\
\hline 20 & 21 & 0 & 0 & $\mathrm{Y}$ & DDI $80 / 40$ & 0 & $\mathrm{~N}$ & $\mathbf{N}$ & $\mathrm{N}$ & DDI $80 / 40$ \\
\hline 21 & 83 & 0 & 0 & $\mathrm{Y}$ & DDI $80 / 40$ & 0 & $\mathbf{N}$ & $\mathrm{N}$ & $\mathbf{N}$ & DDI $80 / 40$ \\
\hline 22 & 41 & 2 & 0.58 & $\mathrm{Y}$ & DDI $80 / 40$ & 1 & $\mathrm{~N}$ & $\mathrm{Y}$ & $\mathrm{N}$ & DDD 60 \\
\hline 23 & $55^{\star}$ & 0 & 0 & $\mathrm{Y}$ & DDI $80 / 50$ & 0 & $\mathrm{~N}$ & $\mathbf{N}$ & $\mathbf{N}$ & DDI $80 / 50$ \\
\hline 24 & 49 & 0 & 0 & $\mathrm{Y}$ & DDI $90 / 50$ & 1 & $\mathrm{~N}$ & $\mathrm{Y}$ & $\mathrm{N}$ & DDI 70 \\
\hline 25 & 63 & 0 & 0 & $\mathrm{~N}$ & DDI $80 / 40$ & 1 & $\mathrm{~N}$ & $\mathbf{N}$ & $\mathrm{N}$ & DDI $90 / 50$ \\
\hline 26 & 74 & 12 & 1.94 & $\mathrm{Y}$ & DDI $85 / 45$ & 4 & Y‡ & $\mathrm{Y}$ & $\mathrm{N}$ & AAI 80 \\
\hline 27 & 54 & 0 & 0 & $\mathbf{N}$ & DDI $90 / 50$ & 0 & $\mathbf{N}^{\top}$ & $\mathbf{N}$ & $\mathrm{N}$ & DDI $90 / 50$ \\
\hline 28 & 93 & 4 & 0.52 & $\mathrm{Y}$ & VVI 70/50 & 0 & $\mathrm{~N}$ & $\mathbf{N}$ & $\mathrm{N}$ & VVI $70 / 50$ \\
\hline 29 & 46 & 2 & 0.53 & $\mathbf{Y}$ & DDI $85 / 45$ & 1 & $\mathrm{~N}$ & $\mathrm{Y}$ & $\mathbf{N}$ & DDI 70 \\
\hline 30 & 26 & 0 & 0 & $\mathrm{Y}$ & DDI $80 / 45$ & 0 & $\mathrm{~N}$ & $\mathbf{N}$ & $\mathrm{N}$ & DDI $80 / 45$ \\
\hline 31 & 51 & 0 & 0 & $\mathrm{~N}$ & DDI $90 / 50$ & 0 & $\mathrm{~N}$ & $\mathrm{~N}$ & $\mathrm{~N}$ & DDI $90 / 50$ \\
\hline 32 & 65 & 0 & 0 & $\mathrm{Y}$ & DDI 80/55 & 1 & $\mathrm{~N}$ & $\mathbf{N}$ & $\mathrm{Y}$ & DDI $70 / 40$ \\
\hline 33 & 77 & 5 & $0 \cdot 78$ & $\mathrm{Y}$ & DDI $80 / 45$ & 0 & $\mathrm{~N}$ & $\mathrm{~N}$ & $\mathbf{N}$ & DDI $80 / 45$ \\
\hline 34 & 76 & 0 & 0 & $\mathbf{N}$ & DDI $85 / 45$ & 0 & $\mathrm{~N}$ & $\mathrm{~N}$ & $\mathrm{~N}$ & DDI $85 / 45$ \\
\hline 35 & 31 & 3 & $1 \cdot 16$ & $\mathrm{Y}$ & DDI $80 / 45$ & 1 & $\mathrm{~N}$ & $\mathrm{Y}$ & $\mathrm{N}$ & DDI 80 \\
\hline 36 & 15 & 0 & 0 & $\mathrm{Y}$ & DDI $75 / 45$ & 0 & $\mathrm{~N}$ & $\mathbf{N}$ & $\mathrm{N}$ & DDI $75 / 45$ \\
\hline 37 & $4^{\star}$ & 0 & 0 & $\mathbf{N}$ & DDI $80 / 50$ & 0 & $\mathrm{~N}$ & $\mathrm{~N}$ & $\mathrm{~N}$ & DDI $80 / 50$ \\
\hline
\end{tabular}

«Patient died during follow up; tpatient developed pacemaker syndrome; $¥ 2$, ventricular lead fracture. FU, follow up; S-fu-n, number of episodes of syncope after implantation; S-freq-fu, frequency of syncope after implantation (episodes/year); PSfu, pre-syncope after implantation; Y, yes; N, no; Mode-1, initial mode and programming; Mode-c, number of mode changes during follow up; Mode-c-t, mode change for technical reason; Mode-c-s, mode change for continuing symptoms; Mode-c-np, mode change for nocturnal pacing, Mode-final, present pacemaker mode and programming.

implantation, findings of investigations before implantation, implantation details, symptoms after implantation, and changes in pacemaker mode were obtained from a review of the hospital notes, pacemaker records, and supplemented where necessary by additional patient interview.

ANALYSIS OF DATA

Data are provided for the entire group and for subgroups with respect to outcome after

Table 4 No syncope compared with syncope after implantation of pacemaker

\begin{tabular}{|c|c|c|c|}
\hline & No syncope & Syncope & p Value \\
\hline $\begin{array}{l}\text { Clinical data: } \\
\text { No (\%) } \\
\text { Mean age (y) } \\
\text { Male sex (\%) } \\
\text { Syncope-no } \\
\text { Syncope-freq } \\
\text { Prodrome (\%) } \\
\text { Injury (\%) }\end{array}$ & $\begin{array}{l}23(62) \\
56(16) \\
14(61) \\
11 \cdot 9(18 \cdot 2,4) \\
3 \cdot 4(5 \cdot 5,1 \cdot 4) \\
16(70) \\
5(22)\end{array}$ & $\begin{array}{l}14(38) \\
69(11 \cdot 2) \\
7(50) \\
19 \cdot 6(29 \cdot 8,6) \\
4 \cdot 5(5 \cdot 5,2 \cdot 5) \\
13(93) \\
6(43)\end{array}$ & $\begin{array}{l}-\bar{p}<0.02^{\star} \\
\text { NSt } \\
\text { NS } \ddagger \\
\text { NS } \\
\text { NSt } \\
\text { NSt }\end{array}$ \\
\hline $\begin{array}{l}\text { Electrocardiogram: } \\
\text { Normal (\%) } \\
\text { SB (\%) }\end{array}$ & $\begin{array}{r}15(65) \\
4(17)\end{array}$ & $\begin{array}{l}6(43) \\
5(36)\end{array}$ & $\begin{array}{l}\text { NSt } \\
\text { NSt }\end{array}$ \\
\hline $\begin{array}{l}\text { Tilt data: } \\
\text { Time Pos } \\
\text { HRs } \\
\text { HRmax } \\
\text { HRe } \\
\text { Asystole (\%) } \\
\text { BPSYSs } \\
\text { BPDIAs }\end{array}$ & $\begin{array}{c}20 \cdot 1(12 \cdot 5) \\
66 \cdot 8(9) \\
85(17 \cdot 8) \\
31(20 \cdot 1) \\
7(30) \\
137(21) \\
82 \cdot 8(13 \cdot 4)\end{array}$ & $\begin{array}{c}26 \cdot 4(14 \cdot 1) \\
65 \cdot 5(14 \cdot 2) \\
79 \cdot 1(13 \cdot 3) \\
29 \cdot 6(14 \cdot 9) \\
4(29) \\
152(28) \\
79 \cdot 3(15 \cdot 4)\end{array}$ & $\begin{array}{l}N^{\star} \\
N^{\star} \\
N^{\star} \\
N^{\star} \\
N^{\star} \\
N^{\star} \\
N^{\star} \\
\text { SS }^{\star}\end{array}$ \\
\hline $\begin{array}{l}\text { Follow up data: } \\
\text { FU (months) } \\
\text { S-fu-n } \\
\text { S-freq-fu }\end{array}$ & $\begin{array}{l}46 \cdot 3(24 \cdot 1) \\
0 \\
0\end{array}$ & $\begin{array}{l}56 \cdot 5(22 \cdot 8) \\
3.3(2 \cdot 8,2 \cdot 5 \S) \\
0.7(0.4,0.64 \S)\end{array}$ & $\begin{array}{l}\mathrm{NS}^{\star} \\
-\end{array}$ \\
\hline $\begin{array}{l}\text { Pacemaker implant: } \\
\text { Dual chamber mode (\%) } \\
\text { DDI-hysteresis (\%) } \\
\text { Lower rate }\end{array}$ & $\begin{array}{l}22(96) \\
21(91) \\
48 \cdot 3(7 \cdot 2)\end{array}$ & $\begin{array}{l}13(93) \\
10(71) \\
49 \cdot 6(6 \cdot 6)\end{array}$ & $\begin{array}{l}\text { NSt } \\
\text { NSt } \\
\text { NS* }\end{array}$ \\
\hline
\end{tabular}

$\star^{\star}$ t test. $+\chi^{2}$ test with Yates correction. $¥ M a n n-W h i t n e y ~ U$ test. Imedium. Time pos, time (min) at which tilt test was positive; Asystole, sinus pause $\geqslant 4 \mathrm{~s}$ at syncope; Lower rate, basic or hysteresis trigger rate programmed after implantation; SD or percentages in parentheses. For other abbreviations see footnotes to tables 1,2 and 3 . implantation (syncope $v$ no syncope, and abolition of all symptoms $v$ continuing symptoms or syncope). The significance of asystole during tilt (defined as a sinus pause of at least four seconds) with respect to outcome after implantation, was also assessed in view of the emphasis placed on this finding in previous work. ${ }^{15} 1821$

The unpaired $t$ test and $\chi^{2}$ test with Yates correction were used to assess differences between the defined subgroups except for assessment of differences in total syncope and frequency of syncope. These data were not normally distributed and were compared with the Mann-Whitney $U$ test. A $p$ value of $<0.05$ was considered significant.

\section{Results}

Thirty seven patients fulfilled the inclusion and exclusion criteria and form the study group.

\section{PATIENT CHARACTERISTICS BEFORE} IMPLANTATION

The patients received permanent pacemakers between 1985 and 1991. They represented $3.4 \%(37 / 1073)$ of all new patients paced in our unit during this period. The group was $57 \%$ male with a mean age of 61 (SD 15.6, range 33-89) years. They had a mean of 14.8 (SD $23 \cdot 2$, median 6 , range 1-110) syncopal episodes at a mean frequency of 3.8 (SD 5.5 , median 2 , range $0 \cdot 2-25$ ) syncopes/year. $78 \%$ reported prodromal symptoms and $30 \%$ had sustained injury. Coexistent cardiovascular disease was present in $26 \%$ (hypertension 
Table 5 No syncope or presyncope compared with syncope or presyncope after implantation of pacemaker.

\begin{tabular}{|c|c|c|c|}
\hline & $\begin{array}{l}\text { No syncope } \\
\text { or presyncope }\end{array}$ & $\begin{array}{l}\text { Syncope } \\
\text { or presyncope }\end{array}$ & $p$ Value \\
\hline $\begin{array}{l}\text { Clinical data: } \\
\text { No (\%) } \\
\text { Mean age (y) } \\
\text { Male sex (\%) } \\
\text { Syncope-no } \\
\text { Syncope-freq } \\
\text { Prodrome (\%) } \\
\text { Injury (\%) }\end{array}$ & $\begin{array}{l}10(27) \\
49 \cdot 7(12 \cdot 5) \\
9(90) \\
6(8 \cdot 8,29) \\
2 \cdot 3(3 \cdot 4,19) \\
8(80) \\
1(10)\end{array}$ & $\begin{array}{l}27(73) \\
65 \cdot 2(14 \cdot 6) \\
12(44) \\
18(26,6 \S) \\
4 \cdot 3(6,2 \S) \\
21(78) \\
10(37)\end{array}$ & $\begin{array}{l}-<0.01 \star \\
<0.05 \dagger \\
<0.02 \ddagger \\
\text { NS } \\
\text { NS† } \\
\text { NSt }\end{array}$ \\
\hline $\begin{array}{l}\text { Electrocardiogram: } \\
\text { Normal }(\%) \\
\text { SB (\%) }\end{array}$ & $\begin{array}{l}6(60) \\
2(20)\end{array}$ & $\begin{array}{r}15(56) \\
7(26)\end{array}$ & $\begin{array}{l}\text { NSt } \\
\text { NSt }\end{array}$ \\
\hline $\begin{array}{l}\text { Tilt data: } \\
\text { Time pos } \\
\text { HRs } \\
\text { HRmax } \\
\text { HRe } \\
\text { Asystole (\%) } \\
\text { BPSYSs } \\
\text { BPDIAs }\end{array}$ & $\begin{array}{c}17 \cdot 9(13 \cdot 9) \\
66 \cdot 7(11) \\
86 \cdot 6(16 \cdot 7) \\
32 \cdot 6(21 \cdot 6) \\
3(30) \\
136(17) \\
84 \cdot 3(11 \cdot 8)\end{array}$ & $\begin{array}{c}24 \cdot 2(12 \cdot 9) \\
66 \cdot 1(11 \cdot 3) \\
81 \cdot 3(16 \cdot 2) \\
29 \cdot 7(17) \\
8(30) \\
146(27) \\
80 \cdot 4(14 \cdot 9)\end{array}$ & $\begin{array}{l}\text { NS }^{\star} \\
\text { NS }^{\star} \\
\text { NS }^{\star} \\
\text { NS }^{\star} \\
\text { NSt } \\
\text { NS }^{\star} \\
\text { NS* }^{\star}\end{array}$ \\
\hline $\begin{array}{l}\text { Follow up data: } \\
\text { FU (months) } \\
\text { S-fu-n } \\
\text { S-freq-fu }\end{array}$ & $\begin{array}{l}47 \cdot 2(27 \cdot 7) \\
0 \\
0\end{array}$ & $\begin{array}{l}51 \cdot 3(22 \cdot 8) \\
1 \cdot 7(2 \cdot 6,1 \emptyset) \\
0 \cdot 4(0 \cdot 5,0 \cdot 2 \S)\end{array}$ & $\frac{N^{\star}}{-}$ \\
\hline $\begin{array}{l}\text { Pacemaker implant: } \\
\text { Dual chamber mode (\%) } \\
\text { DDI hysteresis (\%) } \\
\text { Lower rate }\end{array}$ & $\begin{array}{c}10(100) \\
9(90) \\
47 \cdot 5(5 \cdot 9)\end{array}$ & $\begin{array}{l}25(93) \\
22(81) \\
49 \cdot 3(5 \cdot 9)\end{array}$ & $\begin{array}{l}\text { NSt } \\
\text { NSt } \\
\text { NS* }\end{array}$ \\
\hline
\end{tabular}

Footnotes as for table 4.

$16 \%$, angina $11 \%$, previous inferior myocardial infarction 5\%, atrial fibrillation 3\%). No patient had orthostatic hypotension or clinical evidence of neurological disease. Two patients were taking $\beta$-blockers, two were taking diuretics, and one was taking a calcium antagonist. No other patient was taking cardiovascular medications at the time of investigation.

INVESTIGATIONS BEFORE IMPLANTATION

Conventional investigations and an invasive electrophysiological study had failed to provide a diagnosis in any patient. A non-diagnostic abnormality on the 12 lead resting electrocardiogram was present in $46 \%$ (sinus bradycardia $24 \%$, left anterior fascicular block $8 \%$, inferior myocardial infarction $5 \%$, first degree atrioventricular block $3 \%$, right

Table 6 Asystole compared with absence of asystole during tilt

\begin{tabular}{|c|c|c|c|}
\hline & Asystole $(\geqslant 4 s)$ & No asystole & p Value \\
\hline $\begin{array}{l}\text { Clinical data: } \\
\text { No (\%) } \\
\text { Mean age (y) } \\
\text { Male sex (\%) } \\
\text { Syncope-no } \\
\text { Syncope-freq } \\
\text { Prodrome (\%) } \\
\text { Injury (\%) }\end{array}$ & $\begin{array}{l}11(30) \\
58 \cdot 8(17) \\
7(64) \\
11 \cdot 5(17 \cdot 7,4 \S) \\
3(3 \cdot 5,29) \\
9(82) \\
2(18)\end{array}$ & $\begin{array}{l}26(70) \\
62(15 \cdot 2) \\
14(54) \\
16 \cdot 2(25 \cdot 4,6 \S) \\
4 \cdot 2(6 \cdot 1,1 \cdot 7 \S) \\
20(77) \\
9(35)\end{array}$ & $\begin{array}{l}\overline{N S} \\
\text { p < } 0.05 \\
\text { NS } \ddagger \\
\text { NS } \\
\text { NSt } \\
\text { NSt }\end{array}$ \\
\hline $\begin{array}{l}\text { Electrocardiogram: } \\
\text { Normal (\%) } \\
\text { SB (\%) }\end{array}$ & $\begin{array}{l}7(64) \\
2(18)\end{array}$ & $\begin{array}{r}14(54) \\
7(27)\end{array}$ & $\begin{array}{l}\text { NSt } \\
\text { NSt }\end{array}$ \\
\hline $\begin{array}{l}\text { Tilt data: } \\
\text { Time pos } \\
\text { HRs } \\
\text { HRmax } \\
\text { HRe } \\
\text { Asystole (\%) } \\
\text { BPSYSs } \\
\text { BPDIAs }\end{array}$ & $\begin{array}{c}22(14 \cdot 9) \\
63(15 \cdot 9) \\
80(20 \cdot 6) \\
7(4 \cdot 2) \\
11(100) \\
134 \cdot 2(18 \cdot 8) \\
76 \cdot 4(11 \cdot 8)\end{array}$ & $\begin{array}{c}22 \cdot 7(12 \cdot 9) \\
67 \cdot 7(8 \cdot 4) \\
83 \cdot 9(14 \cdot 4) \\
40 \cdot 4(11) \\
0 \\
146 \cdot 7(26 \cdot 5) \\
83.6(14.6)\end{array}$ & $\begin{array}{l}\text { NS }^{\star} \\
\text { NS }^{\star} \\
\text { NS }^{\star} \\
\overline{-} \\
\text { NS }^{\star} \\
\text { NS }^{\star}\end{array}$ \\
\hline $\begin{array}{l}\text { Follow up data: } \\
\text { FU (months) } \\
\text { S-fu-n } \\
\text { S-freq-fu }\end{array}$ & $\begin{array}{l}38 \cdot 7(26 \cdot 7) \\
1 \cdot 1(1 \cdot 8,0 \S) \\
0.3(0 \cdot 4,0 \S)\end{array}$ & $\begin{array}{l}55(21 \cdot 3) \\
1 \cdot 3(2 \cdot 6,05) \\
0 \cdot 3(0 \cdot 5,05)\end{array}$ & $\begin{array}{l}\text { NS* } \\
\text { NS } \ddagger \\
\text { NS }\end{array}$ \\
\hline $\begin{array}{l}\text { Pacemaker implant: } \\
\text { Dual chamber mode (\%) } \\
\text { DDI hysteresis (\%) } \\
\text { Lower rate }\end{array}$ & $\begin{array}{c}11(100) \\
8(73) \\
50 \cdot 1(7)\end{array}$ & $\begin{array}{l}24(92) \\
23(88) \\
47 \cdot 9(6 \cdot 8)\end{array}$ & $\begin{array}{l}\text { NSt } \\
\text { NSt } \\
\text { NS* }\end{array}$ \\
\hline
\end{tabular}

Footnotes as for table 4. bundle branch block $3 \%$, atrial fibrillation $3 \%$, and a non-specific repolarisation abnormality $3 \%$ ).

Table 1 shows the clinical characteristics and 12 lead resting electrocardiographic findings before implantation.

RESULTS OF TILT TEST

A total of 60 tilt tests were performed before implantation, $78 \%$ of which were positive. The first tilt was positive in $86 \%$ of patients, and $100 \%$ were positive after three tilt tests. Discordant outcomes of tilt tests were present in $22 \%$ of patients. The mean time to syncope in the positive tilt tests was 22.5 (SD $13 \cdot 3$, median 21 , range $1-55$ ) minutes. The mean heart rate at syncope was 30.5 (SD $18 \cdot 1$, median 33 , range $1-59$ ) beats/min and $30 \%$ of patients developed asystole (sinus pause of at least four seconds) during one or more tilts.

Table 2 shows the tilt test results.

PACEMAKER MODE AND SELECTION OF IMPLANT PROGRAMME

Thirty five patients (95\%) had dual chamber pacemakers implanted. Two patients (one with atrial fibrillation) received VVI pacemakers. Of the dual chamber systems 31 $(84 \%)$ were initially programmed DDI with rate hysteresis, and four were programmed DDD (three units did not provide DDI).

\section{FOLLOW UP}

Patients have been followed up for a mean of 50.2 (SD 23.9, median 51, range 4-93) months. A total of 46 syncopal episodes in the 37 patients occurred after implantation compared with 570 expected episodes (calculated by the sum of the frequencies of syncope before implantation $\times$ duration of follow up for each patient). Twenty three patients $(62 \%)$ have remained free of further syncope and $10(27 \%)$ have remained completely symptom free. Of the 14 patients with recurrent syncope after implantation, 10 report reduced rates of syncope. Therefore 89\% (33/37 patients) improved after implantation. One patient died 4 months after implantation of a myocardial infarction confirmed at postmortem examination. One patient died at 21 months of follow up of a stroke, and a third patient died of cancer 55 months after implantation.

Eighteen of the 37 patients have required pacemaker mode or programme changes. The important indications for such changes were further syncope or presyncope (12 patients) and symptomatic nocturnal pacing (nine patients). One patient was reprogrammed AAI from DDI for pacemaker syndrome and another AAI after displacement of a ventricular lead.

Table 3 shows the follow up data including the initial and current pacemaker mode and programming.

\section{COMPARISONS OF SUBGROUP}

Three subgroup comparisons were performed (no syncope after implantation $v$ further 
syncope after implantation, no syncope or presyncope after implantation $v$ further syncope or presyncope after implantation, and asystole during tilt test $v$ absence of asystole during tilt test).

A younger age at implantation, male sex, and fewer episodes of syncope before implantation were found in the group who had remained free of symptoms after pacing, compared with the group that had continued to experience symptoms. Only a younger age, however, was found in the group that remained free of syncope compared with the group that had not. There was no difference in investigations or tilt test results before implantation, including asystole, between the outcome groups. There were no differences in the pacing mode at implantation, or in programming between the outcome groups.

Tables 4-6 show subgroup data with statistical analysis.

\section{Discussion}

The 37 patients included in this study had no conventional indication for permanent pacing. Clinical evaluation, conventional investigation, and invasive electrophysiological tests had failed to explain their syncope. On the basis of these negative findings, the severity of their presentation and positive cardioinhibitory vasovagal syncope during tilt tests, they were classified as having cardioinhibitory malignant vasovagal syndrome and received permanent pacemakers. Over a mean follow up of more than 4 years none has shown an alternative reason for syncope and collectively the patients have experienced a considerable reduction in the frequency of syncope and symptoms.

The age and sex distributions of these patients were similar to those of all patients tilted for unexplained syncope in our unit over the same period (366 patients, mean (SD) age $61.6(17.6)$ years, $56 \%$ men), and was similar to the tilt positive patients in the series of Raviele et al (mean (SD) age 60 (16) years, $67 \% \mathrm{men}$ ), in which the investigations before tilt testing and tilt test method were similar to our own. ${ }^{9}$ When compared with patients defined as having tilt positive cardioinhibitory vasovagal syndrome in series using different selection criteria and tilt methods, our patients are somewhat older. ${ }^{6-822}$ Despite this difference these patients are probably typical of a syndrome that represents an important proportion of all patients presenting with unexplained syncope. ${ }^{459}$

In $95 \%$ of our patients dual chamber pacing was used in an attempt to maximise haemodynamic benefit during pacing, and minimise the possibility of pacemaker syndrome by maintaining atrioventricular synchrony. ${ }^{23}$ Eighty four per cent of our patients were paced in DDI mode ${ }^{24}$ to obviate the possibility of pacemaker mediated tachycardia, as during a vasovagal episode retrograde atrioventricular conduction may be maintained. ${ }^{25}$ Rate hysteresis was pro- grammed so that under normal conditions patients remained in sinus rhythm. The initial pacing and hysteresis rates were programmed empirically in line with our evolving experience of pacing patients with carotid sinus syndrome, ${ }^{2326}$ taking into account the severity of the bradycardia during tilt tests. When patients had recurrent symptoms or syncope the reprogramming strategy involved an increase in hysteresis rate, or pacing rate, or both.

Subgroup analysis of the data was undertaken in an attempt to identify characteristics before implantation or pacing variables that predicted a more favourable outcome. Apart from the younger age at implantation in patients who remained free of syncope, no other important differences were identified.

\section{THE ROLE OF TILT TESTS IN PATIENT} SELECTION

The use of prolonged head up tilt tests in the diagnostic evaluation of unexplained syncope to identify patients with a predisposition to vasovagal syncope has become increasingly accepted. ${ }^{4-121416182022}$ Furthermore the test provides a haemodynamic profile of the vasovagal decompensation that allows two broad groups of patients to be separated, those with significant bradycardia as well as hypotension at syncope, cardioinhibitory vasovagal syncope-the classical vasovagal syncope described by Lewis ${ }^{27}$-and those with predominant hypotension, vasodepressor vasovagal syncope..$^{520}$ This distinction lacks both an aetiological explanation and a precise definition, but may be important in the choice of treatment. 56920

On the basis of the outcome of the tilt test we implanted pacemakers in patients who developed any degree of bradycardia rather than restrict pacing to patients with the most severe cardioinhibition or asystole as have other centres. ${ }^{91518}$ Interestingly, retrospective analysis of our patients does not suggest a greater impact of pacing treatment on those with tilt induced asystole compared with those who developed lesser degrees of bradycardia. It is possible that whereas tilt testing enables recognition of subjects with vasovagal syncope, the nature of the artificial tilt induced episodes are poor reflections of the clinical vasovagal episodes. Another interpretation of this finding is that the absolute level of cardioinhibition is less important, with respect to pacing efficacy, than the rate of development of bradycardia and its temporal relation to the fall in blood pressure. We have modified our method of tilt test to obtain continuous heart rate and non-invasive blood pressure data, thus enabling a more detailed classification of the events occurring during syncope. ${ }^{28}$ It is possible that this improved method will allow better selection of patients for pacing (fig 1).

\section{EFFICACY OF PACING IN CARDIOINHIBITORY} VASOVAGAL SYNDROME

It has been argued that the vasodepressive element of vasovagal syncope is largely 
Figure 1 (A) Rapid fall in heart rate during cardioinhibitory vasovagal syncope. The heart rate fell from 104 beats/min to asystole over $12.6 \mathrm{~s}$.

Permanent pacing may

have an important role in the management of patients with this type of cardioinhibition.

(B) Gradual fall in heart rate during

cardioinhibitory vasovagal syncope. The heart rate fell from 87 to 34 beats/min over 183.2 s. Permanent pacing may have a more limited role in the

management of patients

with this type of

cardioinhibition.

$T$, tilt; $S$, symptoms

$F$, flat.
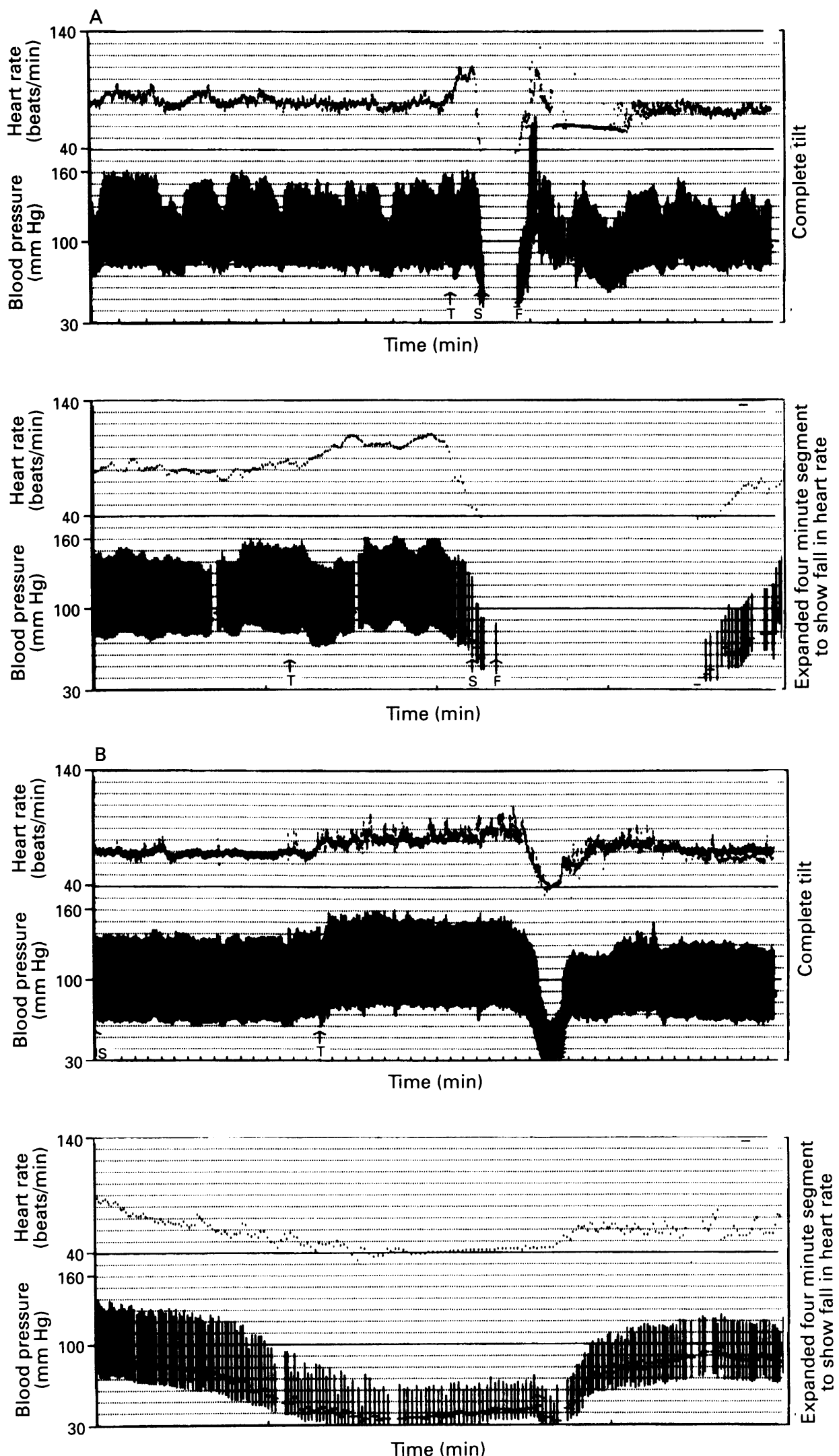

independent of the bradycardia, and thus control of bradycardia would be ineffective in preventing syncope or recurrence of symptoms. ${ }^{102930}$ This opinion is supported by studies in which vagolytic drugs ${ }^{30-33}$ or the use of pacing during induced vasovagal syncope has supported heart rate but failed to obviate hypotension and syncope..$^{29}$

There is, however, contradictory evidence that bradycardia support with pacing treat- ment can ameliorate or abort vasovagal episodes induced by tilt tests. In one study DVI pacing with rate hysteresis aborted vasovagal syncope in five of six patients to give an increase in cardiac index and mean arterial blood pressure compared with an unpaced vasovagal reaction during a previous tilt test. ${ }^{34}$ Grubb et al tested atrioventricular sequential pacing in four patients, with tilt induced asystole, in whom pharmacotherapy had 
Figure 2 Temporary atrioventricular sequential pacing with rate hysteresis during cardioinhibitory vasovagal syncope. The typical haemodynamic response to hysteresis pacing occurred during a vasovagal episode. Although the blood pressure was low, the patient remained conscious and was able to tolerate $a$ further five minutes of tilt. See footnote to fig 1 for abbreviations.
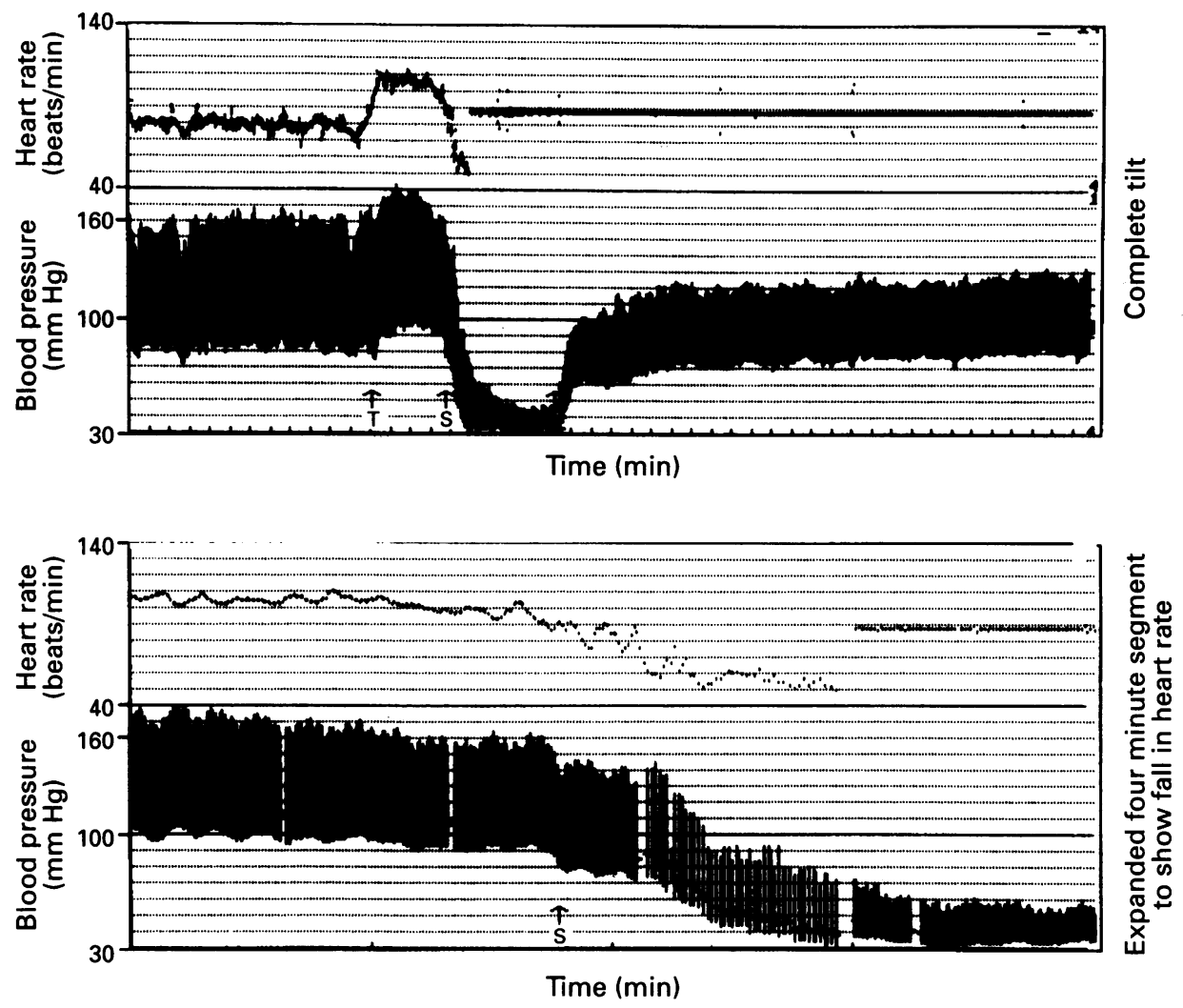

been unsuccessful. ${ }^{18}$ Pacing aborted vasovagal syncope in two and in the others syncope was converted from a sudden dramatic loss of consciousness to a gradual fall in blood pressure over 10 minutes. ${ }^{18}$ These findings are in keeping with our own recent experiences of atrioventricular sequential pacing during tilt induced vasovagal syncope with our updated tilt method (fig 2).

The results of a recently published study, ${ }^{35}$ in which 22 patients with cardioinhibitory vasovagal syncope during tilt underwent a further tilt with temporary pacing, are in line with these earlier studies. ${ }^{1834}$ Patients were paced 20 beats $/ \mathrm{min}$ above their resting rate throughout the tilt test. In the tilt test before pacing all patients were syncopal, but with pacing only five developed syncope. Of the remaining 17 all but two developed vasovagal reactions but remained conscious. This result is remarkably similar to that of Fitzpatrick $e t$ $a l,{ }^{34}$ although the conclusions reached are very different. Whereas Fitzpatrick et al conclude that the modification of the vasovagal episode during pacing could translate into a clinical advantage with permanent pacing, Sra et al dismiss pacing as an ineffective treatment. ${ }^{35}$

There are no reported controlled or randomised studies of permanent pacing for cardioinhibitory vasovagal syncope. There are, however, a few reports of pacing in small uncontrolled series of patients with vasovagal syncope. Abi Samra et al reported that four of six patients treated with pacemakers with tilt positive vasovagal syncope continued to experience syncope although the pacemaker mode and follow up duration were not stated. ${ }^{6}$ Five younger subjects were treated for vasovagal syncope with VVI pacemakers with complete resolution of syncope, in one case over a follow up of nine years. ${ }^{36}$ The four patients in whom atrioventricular sequential pacing aborted or ameliorated tilt induced vasovagal syncope reported by Grubb et al, remained free of syncope during limited follow up. ${ }^{18}$

The relevance of these findings and of our own study are complicated by a lack of data on the natural history of patients with vasovagal syncope, and more specifically, the incidence of syncope or recurrence of symptoms in patients with tilt positive cardioinhibitory malignant vasovagal syncope. Before the use of tilt tests in this context studies of patients with unexplained syncope suggest that $20 \%-50 \%$ of subjects experience further syncope after investigation over a follow up of 20 to 40 months. ${ }^{3738}$ In patients characterised as vasovagal syndrome by tilt tests there is even less information available regarding recurrence of syncope. Four of 15 patients $(27 \%)$ treated with placebo had further syncope over a mean follow up of 9.3 months. ${ }^{39}$ Despite such a limited published experience both an American College of Cardiology/American Heart Association (ACC/AHA) Task Force and a British Pacing and Electrophysiology Group Working Party have recently published recommendations for pacemaker prescription. These include cardioinhibitory malignant vasovagal syncope as an indication, ${ }^{1940}$ although in the ACC/AHA Task Force report it is a class 2 indication and only after temporary atrioventricular sequential pacing during tilt has been shown to be of benefit.

MEDICAL TREATMENT OF VASOVAGAL SYNCOPE Follow up of patients treated medically for tilt 
positive vasovagal syncope with a variety of drugs $(\beta$-blockers, disopyramide, etilefrine hydrochloride, hyoscine, dihydroergotamine, domperidone, and cafedrine) have been reported. ${ }^{1829} 3941$ These studies used tilt tests after giving drugs to identify suitable agents for longer term treatment. Recurrent syncope occurred in $0 \%-20 \%$ of treated patients over a variable follow up (mean 10.7-21 months). Differences in the selection of patients, methods of tilt test, definitions of positive outcomes, and duration of follow up makes meaningful comparison of these studies with our own impossible. In the only placebo controlled study of medical treatment there was no difference in rate of recurrence of syncope between the two groups. ${ }^{39}$

\section{STUDY LIMITATIONS}

This study is retrospective and uncontrolled. Comparison of rates of syncope before and after pacemaker implantation provides apparently powerful evidence to suggest a massive reduction in the predicted collective syncopal burden of the study group. This interpretation of the results of this study should be treated with caution as patients presenting for investigation of syncope may overestimate their previous symptoms, and rates of syncope in patients with unexplained syncope after investigation are lower than expected irrespective of treatment.

In conclusion any treatment for cardioinhibitory malignant vasovagal syndrome must be regarded as provisional and untested. The efficacy of various treatments remains unproved. This study presents an unconventional treatment strategy in a well defined patient group and suggests a possible role for permanent pacing. Pacing should be evaluated more formally in a prospective controlled randomised study.

MEVP is supported by the Garfield Weston Trust.

1 Silverstein MD, Singer DF, Mulley AG, Thibault GE, Barnett GO. Patients with syncope admitted to medical intensive care units. $\mathscr{F A M A} 1982 ; 248: 1185-9$.

2 Kapoor WN, Karpf M, Wieand S, Peterson JR, Levey GS. A prospective evaluation and follow-up of patients with A prospective evaluation and follow-up of pat

3 Muller T, Roy D, Talajic M, Lemery R, Nattel S, Cassidy D. Electrophysiologic evaluation and outcome of patients with syncope of unknown origin. Eur Heart $\mathcal{f}$ 1991;12:139-43.

4 Fitzpatrick A, Theodorakis G, Vardas P, Kenny RA, Travill CM, et al. The incidence of malignant vasovagal syndrome in patients with recurrent syncope. Eur Hear f 1991;12:389-94.

5 Kenny RA, Ingram A, Bayliss J, Sutton R. Head-up tilt: a useful test for the investig

Lancet 1986;2:1352-5.

Abi-Samra F, Maloney JD, Fouad-Tarazi FM, Castle LW. The usefulness of head-up tilt testing and haemodynamic investigations in the workup of syncope of unknown origin. PACE 1988;11:1202-14

7 Almquist A, Goldenberg IF, Milstein S, Chen M-Y, Chen $\mathrm{X}$, Hansen $\mathrm{R}$, et al. Provocation of bradycardia and hypotension by isoproterenol and upright posture in patients with un

8 Strasberg B, Rechavia E, Sagie A, Kusniec J, Mager A, Sclarovsky S, Agmon J. The head-up tilt table test in patients with syncol

9 Raviele A, Gasparini G, Di Pede F, Delise P, Bonso A, Piccolo E. Usefulness of head-up tilt test in evaluating patients with syncope of unknown origin and negative patents with syncope of unknown origin and negative
10 Akhtar M. Unexplained syncope. PACE 1990;13:1533-4. 11 Explaining syncope. Lancet 1991;338:353-4.

12 Kligfield $\mathrm{P}$. Tilt table for the investigation of syncope: is there nothing simple about fainting. $₹ \mathrm{Am}$ Coll Cardiol 1991;17:131-2.

13 van Lieshout J, Wieling W, Karemaker JM, Eckberg DL. The vasovagal response. Clin Sci 1991;81:575-86.

14 Benditt DG, Remole S, Bailin S, Dunnigan A, Asso A, Milstein S. Tilt table testing for evaluation of neurally mediated (cardioneurogenic) syncope: rationale and proposed protocols. PACE 1991;14:1528-37.

15 Maloney JD, Jaeger FJ, Fouad-Tarazi FM, Morris HH. Malignant vasovagal syncope: prolonged asystole provoked by head-up tilt. Cleve Clin $\mathcal{F}$ Med 1988;55:542-8.

16 Fitzpatrick A, Sutton R. Tilting towards a diagnosis in recurrent unexplained syncope. Lancet 1989;i:658-60.

17 Weissler AM, Warren JV. Vasodepressor syncope. Am Heart $\mathcal{F}$ 1959;57:786-94.

18 Grubb BP, Temesy-Armos P, Moore J, Wolfe D, Hahn $\mathrm{H}$, Elliot L. Head-upright tilt table testing in evaluation and management of the malignant vasovagal syndrome. Am ₹ Cardiol 1992;69:904-8.

19 Guidelines for implantation of cardiac pacemakers and antiarrhythmic devices. ACC/AHA task force report. ๆ Am Coll Cardiol 1991;18:1-13.

20 Fitzpatrick AP, Theodorakis G, Vardas P, Sutton R Methodology of head-up tilt testing in patients with unexplained syncope. $\mathcal{F} \mathrm{Am}$ Coll Cardiol 1991;17: unexplaine

21 Milstein S, Buetikofer J, Lesser J, Goldenberg I, Benditt DG, Gornick C, Reyes WJ. Cardiac asystole: a manifestation of neurally mediated hypotension-bradycardia. $\mathcal{F}$ Am Coll Cardiol 1989;14:1626-32.

22 Sheldon R, Killam S. Methodology of isoproterenol-tilt table testing in patients with syncope. $7 \mathrm{Am}$ Coll Cardiol 1992;19:773-9.

23 Morley CA, Sutton R. Carotid sinus syncope. Int $\mathcal{f}$ Cardiol 1984;6:287-93.

24 Floro J, Castellanet M, Florio J, Messenger J. DDI: a new mode for cardiac pacing. Clin Prog Pacing Electrophysiol $1984 ; 2: 255-63$

25 Fitzpatrick AP, Travill CM, Vardos PE, et al. Recurrent symptoms after ventricular pacing in unexplained syncope. PACE 1990;13:619-24

26 Ahmed R, Guneri S, Ingram A, Chan SL, Travill CM, Sutton R. Double blind comparison of DDI, DDI with rate hysteresis, VVI and VVI with rate hysteresis in symptom control in carotid sinus syndrome. $P A C E$ 1991;14:623,A21.

27 Lewis $\mathrm{T}$. Vasovagal syncope and the carotid sinus mechanism with comments on Gowers's and Nothnagels's syndrome. BMF 1932;1:873-6.

28 Sutton R, Petersen M, Brignole M, Raviele A, Menozzi C, Giani P. Proposed classification for tilt induced vasovagal syncope. Eur $f$ Cardiac Pacing Electrophysiol 1992; 3:180-3.

29 Sra JS, Anderson AJ, Sheikh SH, Avitall B, Tchou PJ, Troup PJ, et al. Unexplained syncope evaluated by electrophysiologic studies and head-up tilt testing. Ann Intern Med 1991;114:1013-9.

30 Goldstein DS, Spanarkel M, Pitterman A, Toltzis R, Gratz E, Epstein S, Keiser HR. Circulatory control mechanisms in vasodepressor syncope. Am Heart $\mathfrak{F}$ 1982;104:1071-5.

31 Barcroft H, Edholm OG, McMichael J, Sharpey-Schafer EP. Posthaemorrhagic fainting. Study by cardiac output and forearm flow. Lancet 1944;1:489-91.

32 Weissler AM, Warren JV, Estes EH, McIntosh HD, Leonard JJ. Vasodepressor syncope. Factors influencing cardiac output. Circulation 1957;15:875-82.

33 Murray RH, Shropshire S. Effect of atropine on circulatory responses to lower body negative pressure and vasodepressor syncope. Aerospace Medicine 1970;41: vasodepr

34 Fitzpatrick AP, Theodorakis G, Ahmed R, Williams T, Sutton $R$. Dual chamber pacing aborts vasovagal syncope induced by head-up 60 degree tilt. PACE 1991 ; 14:13-9.

35 Sra JS, Jazayeri MR, Avitall B, et al. Comparison of cardiac pacing with drug therapy in the treatment of neurocardiogenic (vasovagal) syncope with bradycardia or asystole. N Engl $\mathcal{F}$ Med 1993;328:1085-90.

36 Saphire DW, Casta A. Vagotonia in infants, children, adolescents, and young adults. Int $\mathcal{F}$ Cardiol 1985;9:211-22.

37 Kushner JA, Kou WH, Kadish AH, Morady F. Natural history of patients with unexplained syncope and a nondiagnostic electrophysiology study. $尹 \mathrm{Am}$ Coll Cardiol diagnostic electro

38 Kapoor WN. Evaluation and outcome of patients with syncope. Medicine 1990;69:160-75.

39 Brignole M, Menozzi C, Gianfranchi L, Lolli G, Botton $\mathrm{N}$, Oddone $\mathrm{D}$. A controlled trial of acute and long-term medical therapy in tilt-induced neurally mediated syncope. Am $\mathcal{~ C a r d i o l ~ 1 9 9 2 ; 7 0 : 3 3 9 - 4 2 . ~}$

40 Recommendations for pacemaker prescription for symptomatic bradycardia. BPEG working party report. $B$ Heart $\mathcal{F} 1991 ; 66: 185-91$

41 Milstein S, Buetikofer J, Dunnigan A, Benditt DG Gornick C, Reyes WJ. Usefulness of disopyramide for prevention of upright tilt-induced hypotension-bradycardia. Am f Cardiol 1990;65:1339-44. 